\title{
The Empirical Mode Decomposition-Decision Tree Method to Recognize the Steady-State Visual Evoked Potentials with Wide Frequency Range
}

\begin{abstract}
Background: The empirical mode decomposition (EMD) is a technique to analyze the steady-state visual evoked potential (SSVEP) which decomposes the signal into its intrinsic mode functions (IMFs). Although for the limited stimulation frequency range, choosing the effective IMF leads to good results, but extending this range will seriously challenge the method so that even the combination of IMFs is associated with error. Methods: Stimulation frequencies ranged from 6 to $16 \mathrm{~Hz}$ with an interval of $0.5 \mathrm{~Hz}$ were generated using Psychophysics toolbox of MATLAB. SSVEP signal was recorded from six subjects. The EMD was used to extract the effective IMFs. Two features, including the frequency related to the peak of spectrum and normalized local energy in this frequency, were extracted for each of six conditions (each IMF, the combination of two consecutive IMFs and the combination of all three IMFs). Results: The instantaneous frequency histogram and the recognition accuracy diagram indicate that for wide stimulation frequency range, not only one IMF, but also the combination of IMFs does not have desirable efficiency. Total recognition accuracy of the proposed method was $79.75 \%$, while the highest results obtained from the EMD-fast Fourier transform (FFT) and the CCA were $72.05 \%$ and $77.31 \%$, respectively. Conclusion: The proposed method has improved the recognition rate more than $2.4 \%$ and $7.7 \%$ compared to the CCA and EMD-FFT, respectively, by providing the solution for situations with wide stimulation frequency range.
\end{abstract}

Keywords: Brain-computer interface, decision tree classifier, empirical mode decomposition, frequency recognition, harmonic frequency, steady-state visual evoked potential

\section{Introduction}

Steady-state visual evoked potential (SSVEP) is the brain response to repetition of visual stimulation with a certain frequency. It is certain type of evoked potential that refers to stimulation frequencies above $6 \mathrm{~Hz}$. The dominant frequency of SSVEPs depends on the frequency of flickering source. Due to advantages of SSVEP, such as high-data transfer rate and low-training time, this control signal is widely used in brain-computer interface $(\mathrm{BCI})$ systems..$^{[1-3]}$ Several methodologies had been proposed for frequency recognition in SSVEP-based BCI systems. More specifically, the fast Fourier transform (FFT), power spectral density analysis, canonical correlation analysis (CCA), stability coefficient (SC), minimum energy combination, multivariate synchronization index, and least absolute shrinkage and selection operator are used. ${ }^{[4-6]}$

This is an open access journal, and articles are distributed under the terms of the Creative Commons Attribution-NonCommercial-ShareAlike 4.0 License, which allows others to remix, tweak, and build upon the work non-commercially, as long as appropriate credit is given and the new creations are licensed under the identical terms.

For reprints contact: reprints@medknow.com
The EMD is a pre-processing step for SSVEP feature extraction methods. The method of EMD is an adaptive approach to extract time-frequency information of the signal. In this method, since the signal is decomposed into intrinsic mode functions (IMFs) based on local characteristics of it in time-scale, EMD is useful for processing nonstationary signals such as electroencephalogram (EEG). ${ }^{[7,8]}$ The EMD is a data-driven scheme, and any change in input signal (for example, the existence of noise in the signal) will affect signal decomposition and frequency characteristics of its IMFs. Thus, selecting suitable IMF is one of the important issues after the decomposition of the signal. In studies that use limited frequency range, the effective IMF corresponding to that range has usually been analyzed. For example, the transition from attention-to-rest response has been done by analyzing the $\mathrm{IMF}_{6}$ corresponding to very low frequencies $(0.5-2 \mathrm{~Hz}) \cdot{ }^{[9]}$ Ruan et al. have been applied

\begin{tabular}{l} 
How to cite this article: Sadeghi S, Maleki A. \\
The empirical mode decomposition-decision tree \\
method to recognize the steady-state visual evoked \\
potentials with wide frequency range. J Med Sign \\
Sens 2018; XX:XX-XX. \\
Received: May, 2018. Accepted: August, 2018. \\
\hline
\end{tabular}

Sahar Sadeghi, Ali Maleki

Department of Biomedical Engineering, Semnan University, Semnan, Iran
Address for correspondence: Dr. Ali Maleki, Department of Biomedical Engineering, Semnan University, Semnan, Iran. E-mail: amaleki@semnan.ac.ir

Website: www.jmss.mui.ac.ir DOI: $10.4103 /$ jmss.JMSS_20_18 
$\mathrm{IMF}_{3}$ to analyze two frequencies including 11 and $12 \mathrm{~Hz} \cdot{ }^{[10]}$ In other studies, by assigning a mean instantaneous frequency to each IMF, the IMF with the mean instantaneous frequency closer to stimulation frequencies has applied as effective IMF. In this regard, Wu et al. have focused on $\mathrm{IMF}_{6}$ for the frequency range of $30-35 \mathrm{~Hz}$ by calculating mean instantaneous frequencies. ${ }^{[11]}$

When using wider range of stimulation frequencies, the use of just one IMF will not be sufficient and more IMFs should be analyzed. Huang et al. have focused on $\mathrm{IMF}_{2}$ and $\mathrm{IMF}_{3}$ to apply the frequency range of $34-48 \mathrm{~Hz}$ since the high-frequency SSVEP centralize at these IMFs. ${ }^{[12]}$ In the study done by Wang et al. with SSVEP stimulation frequencies ranged from $9 \mathrm{~Hz}$ to $13 \mathrm{~Hz}$, they have focused on the first four IMFs as their spectra overlapped with stimulation frequencies while discarded the low-frequency components $\left(\mathrm{IMF}_{5-14}\right) \cdot{ }^{[13]}$ Then, since the $\mathrm{IMF}_{3}$ has contained main stimulus frequencies involved, they have combined $\mathrm{IMF}_{3}$ with $\mathrm{IMF}_{1}, \mathrm{IMF}_{2}$, and $\mathrm{IMF}_{4}$ to reconstruct four versions of EMD preprocessed signal: the $\mathrm{IMF}_{3}$; the sum of $\mathrm{IMF}_{3}$ and $\mathrm{IMF}_{4}$; the sum of $\mathrm{IMF}_{2-4}$, and the sum of $\mathrm{IMF}_{1-4}$. They have finally found that the sum of $\mathrm{IMF}_{1-4}$ had the best recognition rate. In our previous study with SSVEP stimulation frequency range of $6-16 \mathrm{~Hz}$, we had used the neural network to classify the recognition results of IMFs. ${ }^{[14]}$

Decision tree (DT) is a popular and powerful method used for classification and prediction purposes. Many previous SSVEP studies have also used this method to classify targets. ${ }^{[15,16]}$ In this paper, we have proposed the DT to classify frequency recognition results of different IMFs. The goal of this paper is to provide an approach for applications with wide range of stimulation frequencies.

\section{Materials and Methods}

\section{Electroencephalogram recording}

EEG signals were recorded using the Bayamed system (EEG V.16.24) at a sampling rate of $250 \mathrm{~Hz}$. Signals were acquired from the surface of the scalp through one electrode placed at $\mathrm{Oz}$, referenced to $\mathrm{AFZ}$, and grounded to the right earlobe. The impedance was kept below $5 \mathrm{~K} \Omega$. All stimuli were presented on a 16" LCD monitor with a refresh rate of $60 \mathrm{~Hz}$ (Samsung NW 1633) using MATLAB and psychophysics toolbox extensions. This toolbox is used to create stimulus with precise frequency and correct timing. The visual stimulus was a white flashing circle with a diameter of $10 \mathrm{~cm}$ rendered at the center of the black background screen.

Six healthy young adults with the age between 21 and 30 years without any known history of BCI experience participated in this study. All subjects were sitting on an armchair in a fixed location, watching a flat screen monitor placed approximately at a distance of $40 \mathrm{~cm}$ from the eye. The nature of the study was fully explained for them before the start of the study and all signed informed consent.
Each subject participated in 10 sessions. In each session, stimulus frequencies were in the range from 6 to $16 \mathrm{~Hz}$ with an interval of $0.5 \mathrm{~Hz}$. Subjects were asked to gaze at the flickering stimulus for $4 \mathrm{~s}$ and then take a $5 \mathrm{~s}$ rest after each trial to avoid visual fatigue caused by flickering. There was 5-min break between two consecutive sessions.

\section{The empirical mode decomposition}

The EMD is the core algorithm of Hilbert-Huang transform that decomposes nonlinear and non-stationary signal to its constructive components (IMFs) with different frequency contents. An IMF must satisfy two conditions: (1) in the whole data of IMF, the number of extrema (maxima and minima) and the number of zero crossings must either equal or differ at most by one and (2) at any point, the mean value of the envelope defined by the local maxima and the envelope defined by the local minima must be zero. By virtue of IMF definition, the decomposition method can simply use envelopes defined by the local maxima and minima separately. Once the extrema are identified, all the local maxima are connected by cubic spline line as the upper envelope. This procedure is repeated for the local minima to produce the lower envelope. Then, the mean value of upper and lower envelop is calculated. The difference between the original data and this mean value is the first component. If this component does not satisfy the definition of IMF, it is substituted with the main signal and enters the cycle as the primary input. Otherwise, this component is as separated IMF and the difference between the main signal and this IMF entered the cycle. ${ }^{[14,17]}$ Due to the overlapping of the stimulation frequency range used in this study with three first IMFs, only these IMFs are extracted as effective IMFs of the signal.

The generalized zero crossing (GZC) method are used to determine the mean instantaneous frequency of an IMF. After defining the local extrema and zero-crossings as critical points, the GZC approach separates each IMF into a series of period based on these points. It estimates the instantaneous frequency of a period by measuring local full and partial wave periods. The instantaneous frequency of each period along the time series can be computed as Eq. 1. To determine the mean instantaneous frequency of an IMF, the ensemble mean of instantaneous frequencies over all time periods in that IMF is calculated as Eq. 2. ${ }^{[1]}$

$$
\begin{aligned}
& f(i)=\frac{1}{12}\left\{\begin{array}{c}
\frac{1}{\mathrm{~T}_{\text {quarter }}(i)}+\left(\frac{1}{\mathrm{~T}_{\text {half }}^{1}(i)}+\frac{1}{\mathrm{~T}_{\text {half }}^{2}(i)}\right)+ \\
\left(\frac{1}{\mathrm{~T}_{\text {full }}^{1}(i)}+\frac{1}{\mathrm{~T}_{\text {full }}^{2}(i)}+\frac{1}{\mathrm{~T}_{\text {full }}^{3}(i)}+\frac{1}{\mathrm{~T}_{\text {full }}^{4}(i)}\right)
\end{array}\right\} \\
& \bar{f}_{G Z C}=\frac{1}{\mathrm{~L}-1} \sum_{i=1}^{\mathrm{L}-1} f(i)
\end{aligned}
$$

Where $f(i)$ is the instantaneous frequency of the $i^{\text {th }}$ time period, $\bar{f}_{\mathrm{GZC}}$ is the mean instantaneous frequency of the IMF and $L$ is the number of critical points denoted as 
$P(i), i=1, \ldots, L$. Assuming that the time period for the $i^{\text {th }}$ critical point is defined as $T(i)=P(i+1)-P(i), \mathrm{T}_{\text {full }}^{k}(i)$ are full periods $(k=1,2,3$ and 4$)$ from $P(I-4+k)$ to $P(i+k)$ covering the $i^{\text {th }}$ time period, $\mathrm{T}_{\text {half }}^{\mathrm{m}}(i)$ are half periods ( $m=1$ and 2 ) from $P(i-2+m)$ to $P(i+m)$, and $T_{\text {quarter }}(i)$ is a quarter period from $P(i)$ to $P(i+1)$.

\section{Decision tree}

In a sequential decision problem, a decision-maker faces a sequence of decisions, nd each decision may impact future decisions. A convenient language to introduce sequential decision problems is through DTs. The most important feature of DT is their capability to break down a complex decision-making process into a collection of simpler decisions, thus providing a solution, which is often easier to interpret. A DT is a connected graph without cycles for illustrating and analyzing a decision, where expected values are calculated alternately. It is composed of the complex of decision nodes which are connected to each other by branches, and branches go down from root nodes until they come to leaf nodes. A DT is started from the top point of it which is called tree root. Indicators are examined in decision nodes, and each result is evaluated in branches. Each branch leads to another decision node or the final leaf node. DTs are used successfully in many diverse areas such as signal classification, character recognition, remote sensing, medical diagnosis, expert systems, and speech recognition, to name only a few. ${ }^{[15,18,19]}$

\section{The proposed method}

Figure 1 shows the block diagram of the proposed method. The length of the processing window has been considered $4 \mathrm{~s}$, according to the recorded signal length during one trial. First, the signal was band-pass filtered from 1 to $40 \mathrm{~Hz}$. Then, effective IMFs (first three IMFs) are extracted from the signal by applying EMD on the filtered signal. In the following, each separate IMF, the combination of two consecutive IMFs and combination of all three IMFs are considered as six conditions. For each condition, two features are extracted. These features include the frequency related to the peak of spectrum and the normalized local energy in this frequency. Eq. 3 defines the normalized local energy where $f_{\max }$ is the frequency related to the peak of the spectrum, $P(f)$ is the power value, and $\Delta \mathrm{f}$ indicates frequency resolution.

$\mathrm{NLE}=\frac{\frac{1}{3} \sum_{\mathrm{i}=-1}^{1} \mathrm{P}\left(\mathrm{f}_{\max }+\mathrm{i} . \Delta \mathrm{f}\right)}{\frac{1}{\mathrm{~N}} \sum_{\mathrm{i}=1}^{\mathrm{N}} \mathrm{P}(\mathrm{i} . \Delta \mathrm{f})}$

Finally, the DT classifier designed based on harmonic detection will be used for data classification.

In the DT design process, at the root node, the maximum frequency ratio of the first two IMFs (IMF1 and IMF2) is investigated. If there is half relation between frequencies, the smaller frequency is accepted as the fundamental stimulation frequency. However, if frequencies do not have harmonic
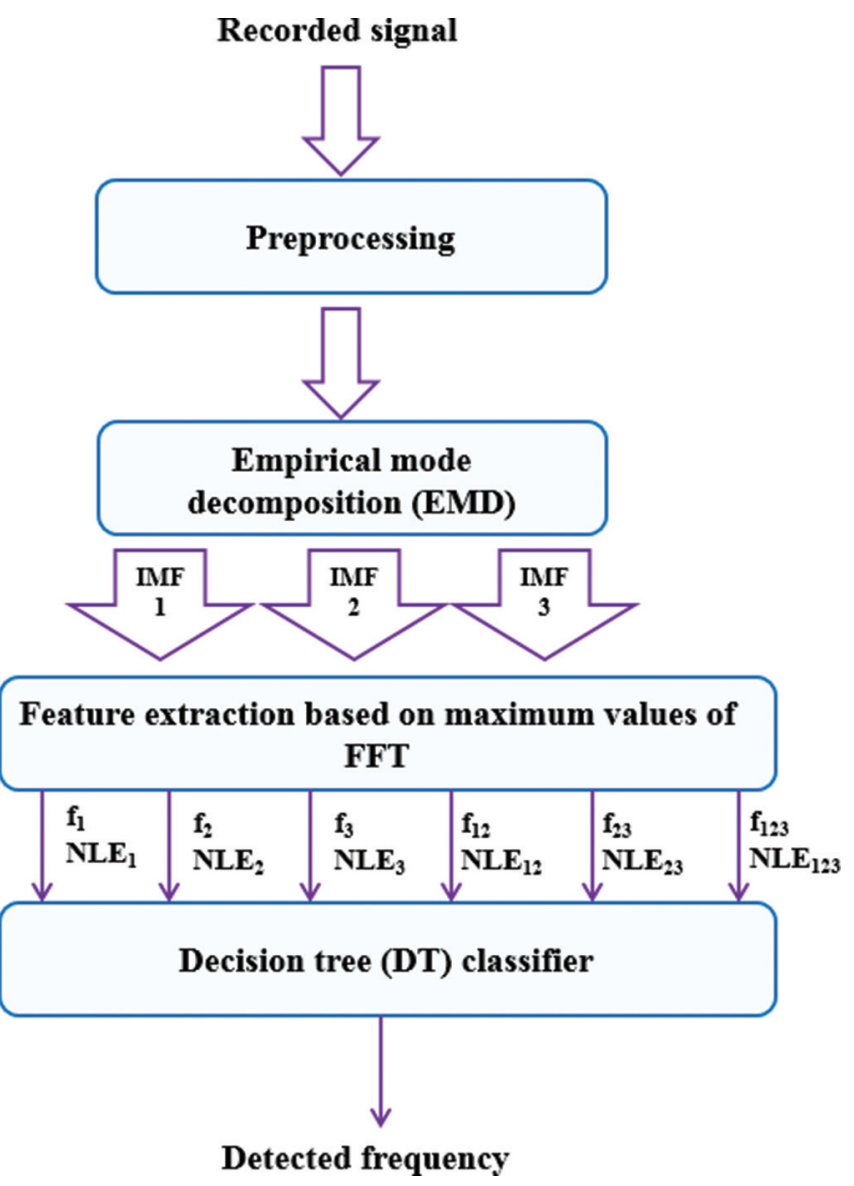

Figure 1: Explanation of the empirical mode decomposition-decision tree model for recognizing steady-state visual evoked potential frequency components, $f_{i}$ denotes the frequency related to the peak of spectrum, and $\mathrm{NLE}_{\mathrm{i}}$ is the normalized local energy in this frequency

relation, this ratio is calculated for the second consecutive IMFs (IMF2 and IMF3). In this step, if the condition is true, the normalized local energy ratio of these IMFs must also be calculated, and the final decision is made from the response of the energy ratio condition. Otherwise, the maximum frequency ratio of the effective IMFs and the combination of IMFs is evaluated step-by-step. In general, in these nodes, if there is a half-relation between frequencies, the smaller frequency is accepted as the fundamental stimulation frequency; otherwise, they go to the next comparison node. Figure 2 shows the proposed classification algorithm.

\section{Results}

Figure 3 illustrates the instantaneous frequency histogram of six conditions (each separate IMF, the combination of two consecutive IMFs and combination of all three IMFs) for six subjects, 10 sessions for each subject and 21 stimulation frequencies for each session. Results of recognition accuracy ${ }^{[14]}$ stimulation frequency are illustrated in Figure 4 for two combinations of two consecutive IMFs. As shown from the figure, the recognition rate of $\mathrm{IMF}_{23}$ has a high value for low stimulation frequencies; however, this rate will gradually decline with increasing frequency. 


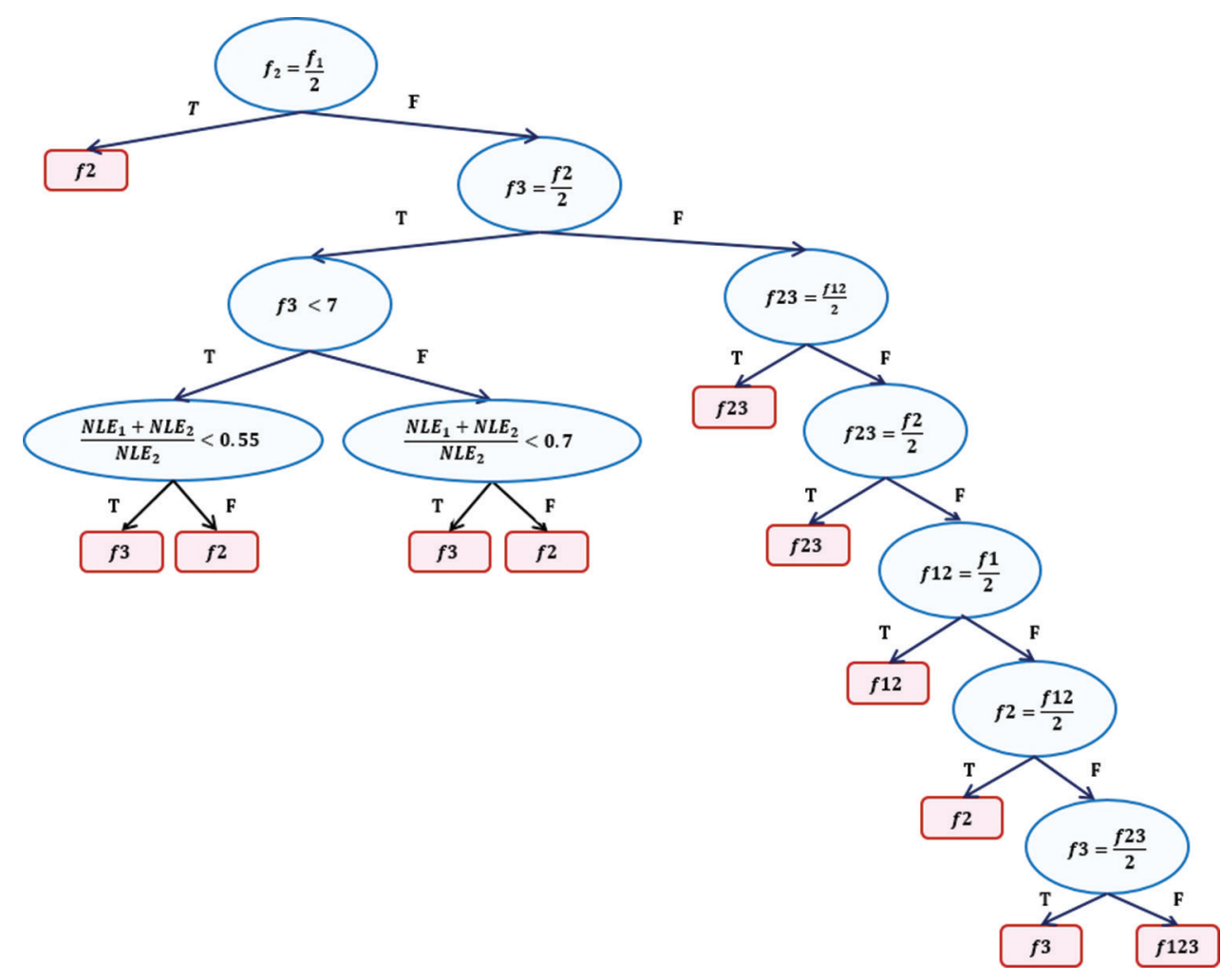

Figure 2: The proposed classification algorithm based on decision tree

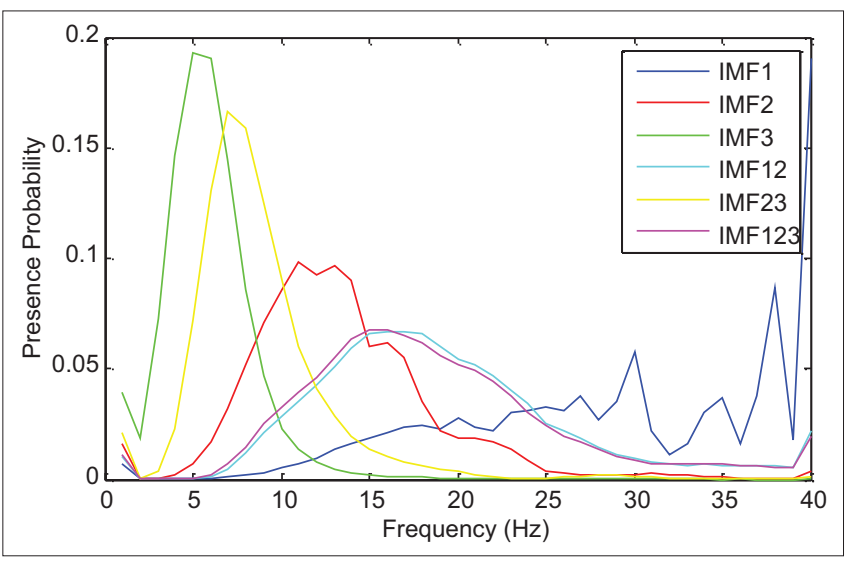

Figure 3: The instantaneous frequency histogram for six-intrinsic mode function-combination conditions

On the other hand, $\mathrm{IMF}_{12}$ associated with good recognition accuracy, only for the middle and upper-frequency ranges.

The recognition accuracy values of the proposed method for each subject were calculated according to the Table 1 . To compare the proposed approach with EMD-based methods used in previous studies, accuracy values of the EMD-FFT (in different conditions) have been computed. To further evaluate the validity of the proposed method, results of the benchmark CCA method (for two cases, choosing one harmonic $(n=1)$ and two harmonics $(n=2)$ in constructing reference signals) have also been reported.

The highest recognition accuracy of the proposed method was obtained by subject 3 with $89.04 \%$ while the lowest value was achieved by subject 4 with $63.80 \%$. The total mean recognition rate was obtained $79.75 \%$ for the proposed method while the maximum corresponding value obtained by six EMD-FFT conditions was $72.05 \%$. The accuracy value for the CCA achieved by choosing one and two harmonics were $76.47 \%$ and $77.31 \%$, respectively.

\section{Discussion}

In the EMD, decomposition method, selecting an IMF based on the proximity with the stimulation frequency range, has optimum performance only for limited stimulation frequency ranges. If we use wider range of frequencies, this selecting method and even the method of IMF combination are not satisfactory. The instantaneous frequency histogram for six IMF combination conditions reveals that for wider range of stimulation frequencies, not only one IMF, but also the combination of IMFs does not have desirable efficiency. Moreover, the results of recognition accuracy also indicate inefficient use of IMF for the selected stimulation frequency range.

\section{Conclusion}

The idea of selecting the desired IMF adaptively based on Decision tree, has improved the recognition rate more than $2.4 \%$ and $7.7 \%$ compared to the CCA and EMD FFT, respectively. Using the proposed method, the utilization of the wide stimulation frequency range has been provided in SSVEP based BCI systems. 


\begin{tabular}{|c|c|c|c|c|c|c|c|c|c|}
\hline \multirow[t]{3}{*}{ Subject } & \multicolumn{9}{|c|}{ Accuracies (\%) } \\
\hline & \multicolumn{6}{|c|}{ EMD-FFT } & \multirow[t]{2}{*}{$\operatorname{CCA}(n=1)$} & \multirow[t]{2}{*}{$\operatorname{CCA}(n=2)$} & \multirow[t]{2}{*}{ Proposed metho } \\
\hline & IMF $_{1}$ & IMF $_{2}$ & $\mathrm{IMF}_{3}$ & $\mathrm{IMF}_{12}$ & $\mathbf{I M F}_{23}$ & IMF1 $_{23}$ & & & \\
\hline 1 & 25.71 & 67.61 & 15.71 & 73.80 & 69.52 & 81.90 & 82 & 78.09 & 82.38 \\
\hline 2 & 22.85 & 54.28 & 18.57 & 59.04 & 56.66 & 60.95 & 68.57 & 72.76 & 72.85 \\
\hline 3 & 41.9 & 74.76 & 14.76 & 76.66 & 80.95 & 80.47 & 85.71 & 85.23 & 89.04 \\
\hline 4 & 36.66 & 44.28 & 11.42 & 57.61 & 48.09 & 57.61 & 62.09 & 63.04 & 63.80 \\
\hline 5 & 38.57 & 50 & 10.95 & 70 & 54.76 & 74.76 & 80.95 & 81.42 & 82.38 \\
\hline 6 & 59.04 & 59.04 & 8.57 & 76.19 & 59.04 & 76.66 & 79.52 & 83.33 & 88.09 \\
\hline Average & 37.45 & 68.57 & 13.33 & 68.88 & 61.5 & 72.05 & 76.47 & 77.31 & 79.75 \\
\hline
\end{tabular}

IMF - Intrinsic mode function; CCA - Canonical correlation analysis; EMD - Empirical mode decomposition; FFT - Fast Fourier transform

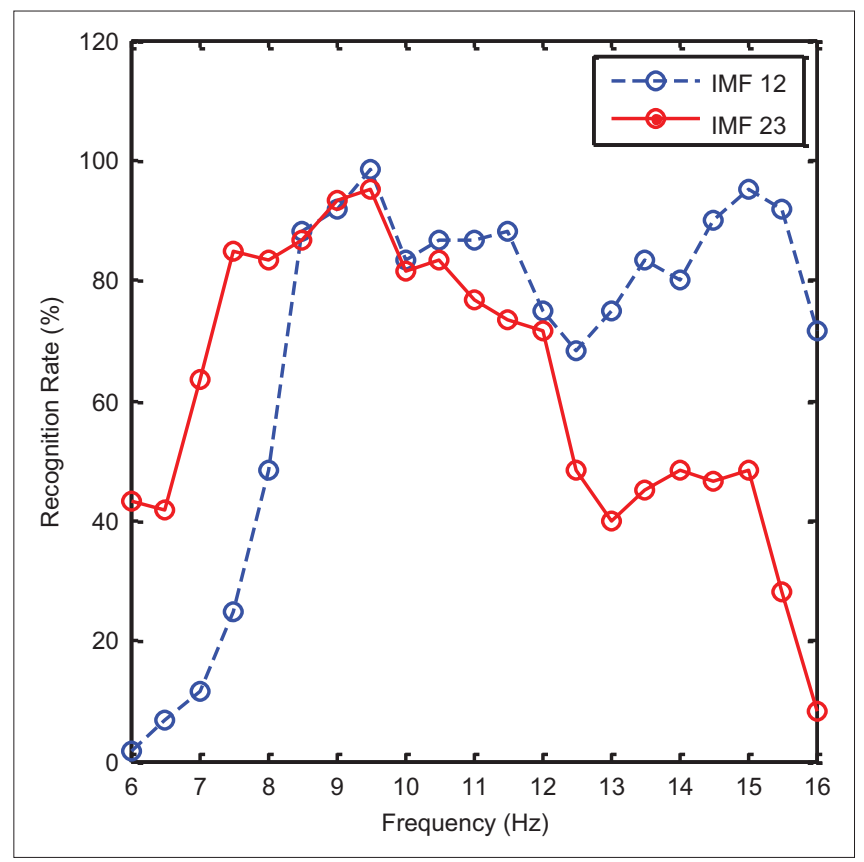

Figure 4: The comparison of recognition accuracy regarding stimulation frequency for two combinations of two consecutive intrinsic mode functions

Financial support and sponsorship

None.

\section{Conflicts of interest}

There are no conflicts of interest.

\section{References}

1. Waytowich NR, Krusienski DJ. Multiclass steady-state visual evoked potential frequency evaluation using chirp-modulated stimuli. IEEE Trans Hum Mach Syst 2016;46:593-600.

2. Movahedi MM, Mehdizadeh A, Alipour A. Development of a brain computer interface (BCI) speller system based on SSVEP signals. J Biomed Phys Eng 2013;3:81-6.

3. Singla R, Khosla A, Jha R. Influence of stimuli color on steady-state visual evoked potentials based BCI wheelchair control. J Biomed Sci Eng 2013;6:1050.

4. Aceves-Fernandez MA, Fernandez-Fraga SM, Pedraza-Ortega JC, Tovar-Arriaga S. EEG Signal analysis methods based on steady state visual evoked potential stimuli for the development of brain computer interfaces: A review. Am J Comput Sci Eng Surv 2016;4:1-18.
5. Liu Q, Chen K, Ai Q, Xie SQ. Review: Recent development of signal processing algorithms for SSVEP-based brain computer interfaces. J Med Biol Eng 2013;34:299-309.

6. Oikonomou VP, Liaros G, Georgiadis K, Chatzilari E, Adam K, Nikolopoulos S, et al. Comparative Evaluation of State-of-the-Art Algorithms for SSVEP-Based BCIs. ArXiv Preprint arXiv: 1602.00904; 2016.

7. Huang NE, Shen Z, Long SR, Wu MC, Shih HH, Zheng Q, et al. The empirical mode decomposition and the Hilbert spectrum for nonlinear and non-stationary time series analysis. Proc R Soc Lond A Math Phys Eng Sci 1998;454:903-95.

8. Williams N, Nasuto SJ, Saddy JD. Evaluation of empirical mode decomposition for event related potential analysis. EURASIP J Adv Signal Proc 2011;1:1-11.

9. Ng CM, Vai MI. Detection of Attention to Rest Transition from EEG Signals with the Help of Empirical Mode Decomposition. Intell Comput Inf Sci 2011;135:66-71.

10. Ruan X, Xue K, Li M. Feature Extraction of SSVEP-Based Brain-Computer Interface with ICA and HHT Method. IEEE $11^{\text {th }}$ World Congress on Intelligent Control and Automation (WCICA); 2014. p. 2418-23.

11. Wu CH, Chang HC, Lee PL, Li KS, Sie JJ, Sun CW, et al. Frequency recognition in an SSVEP-based brain computer interface using empirical mode decomposition and refined generalized zero-crossing. J Neurosci Methods 2011;196:170-81.

12. Huang M, Wu P, Liu Y, Bi L, Chen H. Application and Contrast in Brain-Computer Interface Between Hilbert-Huang Transform and Wavelet Transform. IEEE $9^{\text {th }}$ International Conference In Young Computer Scientists (ICYCS); 2008. p. 1706-10.

13. Huang L, Huang X, Wang YT, Wang Y, Jung TP, Cheng CK, et al. Empirical mode decomposition improves detection of SSVEP. Conf Proc IEEE Eng Med Biol Soc 2013;2013:3901-4.

14. Sadeghi S, Maleki A. The EMD-CCA with Neural Network classifier to recognize the SSVEP frequency. J Biomed Eng 2017;11:914-8.

15. Heidari H, Einalou Z. SSVEP extraction applying wavelet transform and decision tree with bays classification. Int Clin Neurosci J 2017;4:91-7.

16. Bastos TF, Muller SM, Benevides AB, Sarcinelli-Filho M. Robotic wheelchair commanded by SSVEP, motor imagery and word generation. Conf Proc IEEE Eng Med Biol Soc 2011;2011:4753-6.

17. Wang $\mathrm{YH}$, Yeh $\mathrm{CH}$, Young $\mathrm{HW}, \mathrm{Hu} \mathrm{K}$, Lo MT. On the computational complexity of the empirical mode decomposition algorithm. Phys A Stat Mech Appl 2014;400:159-67.

18. Safavian SR, Landgrebe D. A survey of decision tree classifier methodology. IEEE Trans Syst Man Cybern 1991;21:660-74.

19. Kikuti D, Cozman FG, Shirota Filho R. Sequential decision making with partially ordered preferences. Artif Intell 2011;175:1346-65. 


\section{BIOGRAPHIES}

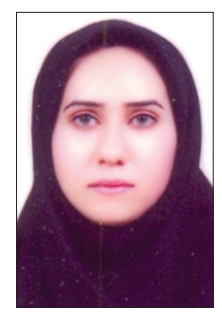

Sahar Sadeghi Sahar Sadeghi received her BSc degree in electrical engineering in 2009 from Babol Noshirvani university of technology, Babol, Iran and her MSc degree in biomedical engineering from Tarbiat Modares University, Tehran, Iran in 2013. She is currently PHD candidate in biomedical engineering at Semnan university. Her research interests are biomedical signal processing and brain-computer interfaces.

Email: sahar.sadeghi@semnan.ac.ir

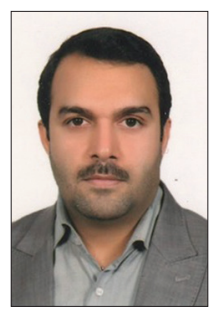

Ali Maleki Ali Maleki received his BSc degree in electrical engineering in 1998 and his MSc (2002) and the PHD (2008) degree in biomedical engineering from Amirkabir university of technology (Tehran Polytechnic), Tehran, Iran. He works as assistant professor at the biomedical engineering department of Semnan university. He is expert in biological system modeling and controlling and biological signal processing.

Email: amaleki@semnan.ac.ir 